Pacific Journal of Mathematics

INVARIANT INTEGRALS OVER A CLASS OF BANACH SPACES 


\title{
INVARIANT INTEGRALS OVER A CLASS OF BANACH SPACES
}

\author{
GERSON B. RoBISON
}

1. Introduction. The main content of this paper is inspired by the theorem of von Neumann [8, p. 92$]$ :

If $S$ is a compact topological group it has a unique invariant integral. ${ }^{1}$

The "invariant integral" may be described as a linear functional $x^{*}$ over $X=C(S)$ which satisfies the following conditions:

(N) $\quad x^{*}(e)=1$ (normalization);

$\left(\mathrm{F}_{1}\right) \quad$ inf $x(t) \geq 0$ implies $x^{*}(x) \geq 0$ (positivity $)^{2}$;

$\left(\mathrm{P}_{2}\right) \quad$ inf $x(t) \geq 0$ and $x \neq 0$ implies $x^{*}(x)>0$ (strict positivity);

(I') for each $x \in X, s \in S$, if the functions $x_{s},{ }^{\prime} x, s_{1} x_{s_{2}}$ are defined by:

$$
\begin{gathered}
x_{s}(t)=x(t s) \\
s^{x}(t)=x(s t) \\
s_{1} x_{s_{2}}(t)=x\left(s_{1} t s_{2}\right),
\end{gathered}
$$

then always:

$$
x^{*}\left(x_{s}\right)=x^{*}\left({ }_{s} x\right)=x^{*}\left({ }_{s_{1}} x_{s_{2}}\right)=x^{*}(x)
$$

(invariance).

The relations $x \longrightarrow x_{s}, x \longrightarrow s x$ are bounded linear transformations on $X$, indexed by $S$. Because of their definition in terms of an underlying topological

\footnotetext{
${ }^{1}$ As stated in the given reference, it is also required that $S$ satisfy the second axiom of countability. However, by use of Ascoli's theorem [3, Chapter X], this can be eliminated.

${ }^{2}$ Unless otherwise stated, inf and sup will always be taken over $S$.
}

Received November 29, 1952.

Pacific J. Math. 4 (1954), 123-150 
group they possess strong properties which enable the construction to go through. We consider here a more general underlying space and a more general class of functions on $S$, together with a semi-group $G$ of bounded linear transformations on this class. The invariance is defined in terms of $G$.

(I) For all $T \in G$, and $x \in X, x^{*}(T x)=x^{*}(x) .^{3}$

Thus the restrictions sufficient for the construction of a unique invariant integral are expressed in terms of the transformations themselves. Since we will be working in terms of linear functionals, it should be noted that $\mathrm{F}_{2}$ implies $\mathrm{F}_{1}$, since $x^{*}(\theta)=0$ for any $x^{*} \in X^{*}$.

2. Notation. $S$ is a topological space. The points of $S$ will be denoted by $t, t_{1}, t^{\prime}$, and so on. $B(S)$ is the collection of bounded, real-valued functions on $S$. Its elements will be denoted by $x, y, z, e$ (the unit function), $\theta$ (the zero function ). Addition in $B(S)$ and scalar multiplication by real numbers are defined linearly:

$$
\begin{gathered}
{[x+y](t)=x(t)+y(t),} \\
{[a x](t)=a[x(t)] .}
\end{gathered}
$$

It has a topology defined by the norm:

$$
\|x\|=\sup |x(t)|
$$

$C(S) \subset B(S)$ is the subspace of continuous bounded functions over $S$. $F$ will be any subset of $B(S)$, subject to certain restrictions as needed; $X$ will be the closed linear manifold generated by $F . X^{*}$ is the space of continuous linear real-valued functionals on $X$ ("dual space", "conjugate space"). Its elements are denoted by $x^{*}, y^{*}$, and so on. Addition and scalar multiplication in $X^{*}$ are defined linearly, as in $X$. Its topology is given by the norm:

$$
\left\|x^{*}\right\|=\sup _{\substack{x \in X \\ x \neq \theta}} \frac{\left|x^{*}(x)\right|}{\|x\|}
$$

For linear functionals, boundedness in the above norm is equivalent to continuity $\left[2\right.$, p. 54]. $B(S), C(S), X$ and $X^{*}$ are all Banach (normed, linear,

${ }^{3}$ Alternatively, this condition may be expressed: " $x *$ is a fixed point under $G^{*}, "$ where $G^{*}=\left\{T^{*}\right\}$, the semigroup of transformations on $X^{*}$, adjoint to $G$, defined as follows: Corresponding to each $T \in G$, a $T^{*} \in G^{*}$ is defined by:

$$
\left[T^{*}\left(x^{*}\right)\right](x)=x^{*}(T x) \text { for each } x \in X \text {. }
$$


complete ) spaces $[2$, p. 53].

$B[X]$ is the algebra of bounded linear transformations on $X$. Addition and scalar multiplication are defined linearly, and

$$
[T V](x)=T[V(x)] \text {. }
$$

It has a topology given by the norm:

$$
\|T\|=\sup _{\substack{x \in X \\ x \neq \theta}} \frac{\|T x\|}{\|x\|}
$$

$G$ will denote a multiplicative semi-group contained in $B[X] . G_{1}$ is the closed convex hull of $G$, that is, the transformations of the form:

$$
V=\sum_{i}^{n} \lambda_{i} T_{i} \quad\left(T_{i} \in G, \lambda_{i} \geq 0, \sum \lambda_{i}=1\right) .
$$

If $G$ is uniformly bounded, that is, if there exists a finite number $k$ such that $\|T\| \leq k$ all $T \in G$, then for any $V \in G_{1}$ :

$$
\|V\|=\left\|\sum \lambda_{i} T_{i}\right\| \leq \sum \lambda_{i}\left\|T_{i}\right\| \leq \max \left\|T_{i}\right\| \text {. }
$$

Hence $G_{1}$ will have the same bound. We shall say in that case, that " $G$ is bounded by $k_{0}$ " $G_{1}$ contains $G$, and $\left(G_{1}\right)_{1}=G_{1}$. For a given $x \in X, K[x]$ is the closure in $B(S)$ of

$$
\left\{y \mid y=V(x), V \in G_{1}\right\},
$$

and $O[x]=\sup x(t)-\inf x(t)$.

3. Note on a result of $\mathbf{J}$. Dixmier. If less is demanded of the invariant integral, a necessary and sufficient condition on $X$ and $G$ for its existence can be obtained by restating and extending a theorem of J. Dixmier [5, pp. 214-215]. In [5], an invariant mean is defined in terms of an underlying topological semigroup, omitting $\mathrm{P}_{2}$ and any requirement of uniqueness, and with $X=C(S)$; the author gives a necessary and sufficient condition for the existence of such a mean, and his theorem may be restated in the terms we are using:

THE OREM 3 (after Dixmier). Let $S$ be any topological space, $X$ any closed subspace of $B(S), G$ as above. Then a necessary and sufficient condition that there exist an $x^{*} \in X^{*}$ satisfying $\mathrm{N}, \mathrm{P}_{1}, \mathrm{I}$, is that for any integer $n$ and any set 
of pairs

$$
\left(x_{i}, T_{i}\right) \quad\left(x_{i} \in X, T_{i} \in G ; i=1,2, \cdots n\right),
$$

we have:

$$
\inf \sum_{1}^{n}\left[x_{i}-T x_{i}\right](t) \leqq 0
$$

Dixmier's proof goes through in this formulation. It should be noted that the condition is quite symmetrical, being equivalent to:

$$
\inf \sum\left[T_{i} y_{i}-y_{i}\right](t)=0 ;
$$

this we obtain by letting $y_{1}=-x_{i}$. The two forms may also be expressed:

$$
\begin{aligned}
& \sup \sum\left[T_{i} x_{i}-x_{i}\right](t)=0, \\
& \sup \sum\left[y_{i}-T_{i} y_{i}\right](t)=0, \text { respectively. }
\end{aligned}
$$

However, this theorem does not ensure uniqueness of the functional so obtained, as the following example will show.

ExAMPLE 3.1. Let $S$ consist of the two points 0,1 , in the discrete topology, $G$ of the identity alone, and

$$
X=C(S)=B(S)
$$

$X$ is then a two-dimensional vector space, with basis $f, g ; f(0)=0, f(1)=1$, and $g(0)=1, g(1)=0$. Any linear functional is determined by its values on $f$ and $g$, by linearity. The Dixmier condition is trivially fulfilled, and so is condition I for any functional. The remaining conditions can be met by assigning the values

$$
\begin{aligned}
& x^{*}(f)=a, \\
& x^{*}(g)=1-a,
\end{aligned}
$$

where $a$ can have any value between 0 and 1.

Furthermore, $\mathrm{P}_{2}$ may be unfulfilled even when $x^{*}$ is unique. 
EXAMPLE 3.2. Let $S$ be the closed interval $0 \leqq t \leqq 1$, with $X=C(S)$. Let $G$ consist of the identity and the transformation $T$ defined by:

$$
[T(x)](t)=x(0) \quad \text { for all } t \in S \text {. }
$$

Thus

$$
T^{2}=T \text { and } T(x)=[x(0)] e .
$$

$G$ satisfies the Dixmier condition since for $t=0$, we have

$$
\sum\left[x_{i}-T_{i} x_{i}\right](t)=0 \text {. }
$$

Hence there is a linear functional $x^{*}$ satisfying $\mathrm{N}, \mathrm{P}_{1}, \mathrm{I}$. But $x$ is completely determined now, since for any $x \in X$ :

$$
x^{*}(x)=x^{*}(T x)=x^{*}([x(0)] e)=[x(0)]\left[x^{*}(e)\right]=x(0),
$$

by N. Thus $x^{*}(x)=0$ for any continuous function if only $x(0)=0$, regardless of how the function behaves elsewhere on $S$. In fact, in this case the functional is identifiable with one on the quotient space $X / M$, where

$$
M=\{x \mid x(0)=0\} .
$$

Since $M$ is a maximal ideal, the quotient space is the field of real numbers [9, Theorem 76].

Finally, the Dixmier criterion refers to all possible finite combinations of equal numbers of functions and transformations, which makes it inconvenient to apply, as may be seen in the examples given in the paper to which we have referred. We have sought conditions which apply to each function and its transformations.

4. The core of the von Neumann construction lies in showing that for each $x \in X$ there is one and only one constant function in $K[x]$. In fact, with the addition of a boundedness condition which occurs naturally in that case, this is sufficient.

Lemma 4.1. For each $x \in X$ and $V \in G_{1}, K[V(x)] \subset K[x]$.

Proof. Let $y$ be in $K[V(x)]$. Then for any $\epsilon>0$ there is a $W$ in $G_{1}$ such that 


$$
\|[W V](x)-y\|<\epsilon .
$$

Hence there is a $V^{\prime} \in G_{1}, V^{\prime}=\mathbb{W} V$, such that

$$
\left\|V^{\prime}(x)-y\right\|<\epsilon \text {. }
$$

Therefore $y$ is in $K[x]$ :

THEOREM 4.1. Suppose that:

(1) $G$ is bounded by $k$;

(2) for any $x \in F$ and $V \in G_{1}, V x \in F$;

(3) constant functions are fixed under $G$ (and hence under $G_{1}$ ), and $F$ contains a constant function $\neq \theta$;

(4) for each $x \in F$ there is just one constant function in $K[x]$.

Then there is a bounded linear functional over $X$ satisfying $\mathrm{N}$ and $\mathrm{I}$. If the constant functions satisfy $\mathrm{P}_{1}$ or $\mathrm{F}_{2}$ (in the obvious meaning) then the functional will satisfy $F_{1}$ or $P_{2}$ over $F$.

Proof. It will be convenient to denote the constant function in $K[x]$, and also its value, by $\bar{x}$. Then we note by (4) and (2) above, and Lemma 4.1, that for each $x \in F$ and $V \in G_{1}$,

$$
\overline{V(x)}=\bar{x} .
$$

Define a function $x^{*}$ over $F$ by

$$
x^{*}(x)=\bar{x}
$$

We assert that for any finite collection $\left\{x_{i}\right\}$ of elements of $F$ and any set of nonzero real numbers $\left\{\lambda_{i}\right\}(i=1,2, \cdots, n)$,

$$
\left|\sum_{1}^{n} \lambda_{i} x^{*}\left(x_{i}\right)\right| \leqq k\left\|\sum_{1}^{n} \lambda_{i} x_{i}\right\| .
$$

To establish $(*)$, we proceed as follows. For $\bar{x}_{1}$, and for any $\epsilon>0$, there is a $V_{1} \in G_{1}$ such that

$$
\left\|V_{1}\left(x_{1}\right)-\bar{x}_{1}\right\|<\epsilon / n k\left|\lambda_{1}\right|
$$


For $\bar{x}_{2}=\overline{V_{1} x_{2}}$ there is a $V_{2}$ such that:

$$
\left\|V_{2}\left[V_{1}\left(x_{2}\right)\right]-\overline{V_{1} x_{2}}\right\|=\left\|\left[V_{2} V_{1}\right]\left(x_{2}\right)-\bar{x}_{2}\right\|<\epsilon / n k\left|\lambda_{2}\right|,
$$

and thus inductively for

$$
\overline{x_{i}}=\left[\overline{\left.V_{i-1} V_{i-2} \cdots V_{1}\right]\left(x_{i}\right)}\right.
$$

there is a $V_{i}$ such that

$$
\left\|\left[V_{i} V_{i-1} \cdots V_{1}\right]\left(x_{i}\right)-\bar{x}_{i}\right\|<\epsilon / n k\left|\lambda_{i}\right| \cdot
$$

Letting $V=V_{n} V_{n-1} \cdots V_{1}$, we get for any $i$ :

$$
\begin{aligned}
\left\|V x_{i}-\bar{x}_{i}\right\| & =\left\|\left[V_{n} V_{n-1} \cdots V_{i+1}\right]\left(\left[V_{i} \cdots V_{1}\right]\left(x_{i}\right)-\bar{x}_{i}\right)\right\| \\
& \leqq\left\|\left[V_{n} \cdots V_{i+1}\right]\right\| \cdot\left\|\left[V_{i} \cdots V_{1}\right]\left(x_{i}\right)-\bar{x}_{i}\right\| \\
& <k \epsilon / n k\left|\lambda_{i}\right|=\epsilon / n\left|\lambda_{i}\right|
\end{aligned}
$$

Hence

$$
\begin{gathered}
\left\|\sum_{1}^{n} \lambda_{i} \bar{x}_{i}\right\|-\left\|\sum_{1}^{n} V\left[\lambda_{i} x_{i}\right]\right\| \leqq\left\|\sum_{1}^{n}\left(V\left[\lambda_{i} x_{i}\right]-\lambda_{i} \bar{x}_{i}\right)\right\| \\
\leqq \sum_{1}^{n}\left|\lambda_{i}\right|\left\|V x_{i}-\bar{x}_{i}\right\|<\epsilon
\end{gathered}
$$

accordingly,

$$
\begin{gathered}
\left|\sum_{1}^{n} \lambda_{i} x^{*}\left(x_{i}\right)\right|=\left\|\sum_{1}^{n} \lambda_{i} \bar{x}_{i}\right\|<\|V\| \cdot\left\|\sum_{1}^{n} \lambda_{i} x_{i}\right\|+\epsilon \\
\leqq K\left\|\sum_{1}^{n} \lambda_{i} x_{i}\right\|+\epsilon .
\end{gathered}
$$

Since $\epsilon>0$ was chosen arbitrarily, our assertion $(*)$ is proved.

Therefore by a theorem of Banach [2, pp.55-56], $x^{*}$ can be extended to be a linear functional over $X$, with 


$$
\left\|x^{*}\right\| \leqq k
$$

Since $F$ contains at least one nonzero constant function, $x^{*}(e)=1$ by linearity. We must show its invariance.

Let

$$
Y=\left\{y \in B(S) \mid y=\sum_{1}^{n} \lambda_{i} x_{i}, x_{i} \in F\right\}
$$

Note that $\bar{Y}=X$. For any $y \in Y$, and any $V \in G_{1}$,

$$
x^{*}[V(y)]=x^{*}\left[V\left(\sum \lambda_{i} x_{i}\right)\right]=\sum \lambda_{i} x^{*}\left[V\left(x_{i}\right)\right]=\sum \lambda_{i} \overline{V x_{i}}=\sum \lambda_{i} \overline{x_{i}}=x^{*}(y) .
$$

Take any $x \in \bar{Y}=X$. Then for any $\epsilon>0$ there is a $y \in Y$ such that

$$
\|y-x\|<\min \left\{\epsilon / 2 k, \epsilon / 2 k^{2}\right\} \text {. }
$$

Therefore

$$
\left|x^{*}(y)-x^{*}(x)\right| \leqq\left\|x^{*}\right\| \cdot\|y-x\|<\epsilon / 2
$$

and

$$
\left|x^{*}[V(y)]-x^{*}[V(x)]\right| \leqq\|x\| \cdot\|V\| \cdot\|y-x\|<\epsilon / 2 .
$$

Hence, since $x^{*}[V(y)]=x^{*}(y)$,

$$
\left|x^{*}[V(x)]-x^{*}(x)\right| \leqq\left|x^{*}[V(x)]-x^{*}[V(y)]\right|+\left|x^{*}(y)-x^{*}(x)\right|<\epsilon .
$$

Since $\epsilon>0$ was chosen arbitrarily,

$$
x^{*}[V(x)]=x^{*}(x) .
$$

The remaining conditions are obviously satisfied.

Lemma 4.2. For the existence of a bounded linear invariant functional $x^{*}$ it is necessary that:

(1) the functional is constant over $K[x]$ for each $x \in X$;

(2) if $x^{*}(e) \neq 0, K[x]$ contains not more than one constant function.

The proof is immediate. 
THEOREM 4.2. If for each $x \in F, K[x]$ contains a constant function, and there exists a bounded linear functional $x^{*}$ over $X$ satisfying $\mathrm{I}$ and $\mathrm{N}$, then it is unique.

Proof. From the preceding lemma, for each $x \in F, K[x]$ contains just one constant function $\bar{x}$. From property $N$ we have, by the same lemma,

$$
x^{*}(x)=x^{*}(\bar{x})=\bar{x} .
$$

Thus the value is completely determined for any $x \in F$ by the value of $\bar{x}$. By linearity it is determined on $Y$; that is, any two functionals $x_{1}^{*}$ and $x_{2}^{*}$ satisfying $\mathrm{N}$ and I must agree at least on $Y$. Take any $x \in X$. Then for any $\epsilon>0$, there is $y \in Y$ such that

$$
\|x-y\|<\min \left\{\epsilon / 2\left\|x_{1}^{*}\right\|, \epsilon / 2\left\|x_{2}^{*}\right\|\right\} .
$$

Then

$$
\left|x_{1}^{*}(x)-x_{1}^{*}(y)\right| \leqq\left\|x_{1}^{*}\right\| \cdot\|x-y\|<\epsilon / 2 \text {. }
$$

Similarly,

$$
\left|x_{2}^{*}(x)-x_{2}(y)\right|<\epsilon / 2 \text {. }
$$

Since $x_{1}^{*}(y)=x_{2}^{*}(y)$, the conclusion follows.

5. In this section we establish conditions sufficient for $K[x]$ to contain at least one constant function. First we establish some properties of a boundedness condition which occurs naturally in the von Neumann construction.

Lemma 5.1. Let $x \in X$ satisfy the condition: ${ }^{4}$

$\left(\mathrm{A}_{1}\right)$ for any $t_{0} \in S$,

$$
\sup _{V \in G_{1}}[V(x)]\left(t_{0}\right)=\sup x(t) \text { and } \inf _{V \in G_{1}}[V(x)]\left(t_{0}\right)=\inf x(t) .
$$

Then:

(1) for any $V \in G_{1}$,

$$
\sup x(t) \geqq \sup [V(x)](t) \text { and } \inf x(t) \leqq \inf [V(x)](t) ;
$$

\footnotetext{
${ }^{4}$ I am indebted to the referee for the simplification of this condition to the above form.
} 
(2) for each $V \in G_{1}, O[V(x)] \leqq O[x]$;

(3) for each $V \in G_{1},\|V(x)\| \leqq\|x\|$;

(4) $V(x)=x$ for all $V \in G_{1}$ if and only if $x$ is a constant function;

(5) for any $y \in K[x]$, inf $x(t) \leqq \inf y(t) \leqq \sup y(t) \leqq \sup x(t)$, and therefore $\|y\| \leqq\|x\|$.

Proof. Conditions (1), (2), and (3) are trivial. If $x$ is a constant function, then

$$
\sup x(t)=\inf x(t)
$$

giving equality throughout in (1), whence $V(x)=x$. Conversely, let $V(x)=x$ all $V \in G_{1}$. Then, for any $t_{0} \in S$,

$$
\sup _{V \in G_{1}}[V(x)]\left(t_{0}\right)=\inf _{V \in G_{1}}[V(x)]\left(t_{0}\right)=x\left(t_{0}\right) .
$$

But by $A_{1}, V$ can be found so that $[V(x)]\left(t_{0}\right)$ approaches as close as we like to either sup $x(t)$ or inf $x(t)$. Hence

$$
\sup x(t)=\inf x(t)=x\left(t_{0}\right) .
$$

Thus (4) is proved. To prove (5), take $y$ in $K[x]$. Let inf $x(t)=c$. Suppose there is a $t_{0} \in S$ such that $y\left(t_{0}\right)=d<c$. Then there is a $V \in G_{\mathbf{l}}$ such that:

$$
|[V(x)](t)-y(t)|<\frac{c-d}{2} \quad \text { for all } t \in S .
$$

Then

$$
[V(x)]\left(t_{0}\right)-d \leqq\left|[V(x)]\left(t_{0}\right)-y\left(t_{0}\right)\right|<\frac{c-d}{2} .
$$

Hence

$$
[V(x)]\left(t_{0}\right)<c=\inf x(t) .
$$

This contradicts $A_{1}$, and therefore

$$
\inf x(t) \leqq \inf y(t) .
$$

Similarly we prove 


$$
\sup x(t) \geqq \sup y(t)
$$

THEOREM 5. Let $S$ be compact, $G$ bounded by $k$. For a given $x$, let $x$ and $V(x)$ be lower (upper) semi-continuous and satisfy $\mathrm{A}_{1}$ for all $V \in G_{1}$. Suppose also that:

$\left(\mathrm{A}_{2}\right) K[x]$ is compact.

Then $K[x]$ contains $a$ 'onstant function $\bar{x}$, such that if $x(t) \geqq 0$ all $t \in S$, then $\bar{x} \geqq 0$. If $x$ and $V(x)$ are lower semi-continuous for all $V \in G_{1}$, with $x(t) \geqq 0$ all $t \in S$, and $x \neq \theta$, then $\bar{x}$ is positive.

We shall first prove the following lemma.

LEMMA 5.2. Under the above hypothesis, (omitting $A_{2}$ ), if $x$ is not a constant function, then there is a $\mathbb{W} \in G_{1}$ such that

$$
\inf x(t)<\inf [\mathbb{W}(x)](t) \leqq \sup [\mathbb{W}(x)](t) \leqq \sup x(t)
$$

( for lower semi-continuity), or

$$
\inf x(t) \leqq \inf [W(x)](t) \leqq \sup [W(x)](t)<\sup x(t)
$$

(for upper semi-continuity), and hence in either case:

$$
O[\mathbb{W}(x)]<O[x]
$$

Proof of Lemma 5.2. Assume $V(x)$ lower semi-continuous for all $V \in G_{1}$. Let

$$
b=\inf x(t)<\sup x(t)=d .
$$

Take $c$ so that $b<c<d$. Then the open set bounded on the left by $c$ contains an image point of $x$. For each $V \in G_{1}$ define the set $M_{v}$ in $S$, open by lower semi-continuity:

$$
M_{v}=\{t \mid[V(x)](t)>c\}
$$

The collection $\left\{M_{v}\right\}, V \in G_{1}$, is an open covering of $S$. For, if $t$ be any point in $S$, by $A_{1}$ there is a $V_{t} \in G_{1}$ such that

$$
\left[V_{t}(x)\right](t)>c ;
$$

$t$ is therefore in $M_{v_{t}}$. Under the compactness of $S$, let 


$$
\left\{M_{v_{i}}\right\} \quad(i=1,2, \cdots, n)
$$

be a finite subcovering. Take

$$
\mathbb{N}=\sum_{1}^{n} \frac{1}{n} V_{i}
$$

Then $W^{\prime} \in\left(G_{1}\right)_{1}=G_{1}$. By Lemma 5.1 we have

$$
\sup [W(x)](t) \leqq \sup x(t)=d .
$$

Also, for any $t_{0}$ and each $V_{i}$,

$$
\left[V_{i}(x)\right]\left(t_{0}\right) \geqq \inf \left[V_{i}(x)\right](t) \geqq \inf x(t)=b .
$$

But for each $t_{0}$ we have, for at least one of the selected $V_{i}$ 's,

$$
\left[V_{i}(x)\right]\left(t_{0}\right)>c .
$$

Hence

$$
[W(x)]\left(t_{0}\right)=\sum_{1}^{n} \frac{1}{n}\left[V_{i}(x)\right]\left(t_{0}\right) \geqq \frac{(n-1) b+c}{n} .
$$

Therefore

$$
\inf [\mathbb{W}(x)](t) \geqq \frac{(n-1) b+c}{n}>b=\inf x(t) .
$$

The proof of the lemma for upper semi-continuous functions is completely analogous.

Proof of Theorem 5. If $x$ is a constant function, $K[x]$ consists of $x$ alone, and the conclusion is satisfied. If $x$ is not constant, let

$$
r_{x}=\inf _{V \in G_{1}} O[V(x)]
$$

We must show that $r_{x}=0$. Let

$$
\left\{V_{i}\right\}
$$$$
(i=1,2, \cdots)
$$

be a sequence in $G_{1}$ such that $O\left[V_{i}(x)\right] \rightarrow r_{x}$. By the compactness of $K[x]$ there is a subsequence $\left\{W_{i}\right\}$ and a $y \in K[x]$ such that 


$$
W_{i}(x) \rightarrow y \text { and } O\left[W_{i}(x)\right] \rightarrow r_{x}
$$

Take any $\epsilon>0$. For $i$ sufficiently large,

$$
\begin{gathered}
\left\|W_{i}(x)-y\right\|<\epsilon / 3, \\
\left|O\left[W_{i}(x)\right]-r_{x}\right|<\epsilon / 3 .
\end{gathered}
$$

From (1), in standard fashion, we get

$$
\begin{aligned}
& \left|\sup y(t)-\sup \left[W_{i}(x)\right](t)\right| \leqq \epsilon / 3, \\
& \left|\inf \left[W_{i}(x)\right](t)-\inf y(t)\right| \leqq \epsilon / 3,
\end{aligned}
$$

whence

$$
\left|O[y]-O\left[\mathbb{W}_{i}(x)\right]\right| \leqq 2 \epsilon / 3
$$

Adding this to the inequality (2) above, we obtain $\left|O[y]-r_{x}\right|<\epsilon_{0}$. Since $\epsilon$ was arbitrary, $O[y]=r_{x}$.

Now assume $r_{x}>0$. Since the convergence of $W_{i}(x)$ to $y$ is uniform, the latter has the same kind of semi-continuity and satisfies $A_{1}$. Hence by Lemma 5.2 there is a $V \in G_{1}$ such that

$$
O[V(y)]<O[y]=r_{x} \text {. }
$$

Let

$$
r_{x}-O[V(y)]=\delta>0 \text {. }
$$

Take $i$ sufficiently large, so that

$$
\left\|y-W_{i}(x)\right\|<\delta / 3 k .
$$

Then

$$
\left\|V(y)-\left[V W_{i}\right](x)\right\| \leqq\|V\| \cdot\left\|y-W_{i}(x)\right\|<\delta / 3
$$

As before, we get

$$
O\left[V W_{i}(x)\right]-O[V(y)] \leqq\left|O\left[V W_{i}(y)\right]-O[V(y)]\right| \leqq 2 \delta / 3 .
$$

Subtracting from equality (3), we get

$$
r_{x}-O\left[V W_{i}(x)\right] \geqq \delta-2 \delta / 3=\delta / 3 .
$$


Therefore

$$
O\left[V W_{i}(x)\right] \leqq r_{x}-\delta / 3<r_{x}
$$

But this contradicts the definition of $r_{x}$, since $V W_{i}$ is in $G_{1}$. Hence $r_{x}=0$, and $y$ is the required function.

Now suppose $x(t) \geqq 0$, for all $t \in S$. Then by Lemma 5.1 ( 5 ),

$$
y=\bar{x} \geqq 0 \text {. }
$$

If also $x$ and $V(x)$ are lower semi-continuous for all $V \in G_{1}$ and $x \neq \vartheta$, then by Lemma 5.2 there is a $W \in G_{1}$ such that

$$
0 \leqq \inf x(t)<\inf [W(x)](t) .
$$

By the foregoing there is a constant function $y=\overline{W(x)}$ in $K[W(x)]$. By Lemma $5.1(5)$,

$$
y(t) \geqq \inf [W(x)](t)>\inf x(t) \geqq 0 \text {. }
$$

By Lemma 4.1, $y \in K[x]$, and this satisfies the last statement of the theorem.

If $S$ is a compact, uniform space ${ }^{5}$ (or a compact Hausdorff, and hence uniformizable space), and $K[x] \subset C(S)$, then $\mathrm{A}_{2}$ may be replaced by a condition which enables us to obtain a suitable equicontinuous family of functions, whereupon Ascoli's theorem may be applied [3, Chapter 10]. This is the situation in von Neumann's construction, where the transforms of $x$ under $G_{1}$ are indeed uniformly equi continuous.

COROLLAR Y 5. Let $S$ be a compact uniform space, II its uniform structure. Then in Theorem 5, if $K[x] \subset C(S), \mathrm{A}_{2}$ may be replaced by:

$\left(\mathrm{A}_{2}^{\prime}\right)$ For each $V \in G_{1}$ there is a $W \in G_{1}$ such that for any $\epsilon>0, t \in S$, and $\psi \in \mathrm{II}$, there is a $\delta>0$ for which, if $O[x]<\delta$ over $\psi(t)$ then $O[W V(x)]<\epsilon$ over $\psi(t)$.

In the proof of the theorem we need only replace the first sequence $V_{i}$ with $W_{i} V_{i}$, the $W_{i}$ 's having been determined in accordance with $A_{2}^{\prime}$. By $A_{1}$ and Lemma 5.1 (2), $O\left[V_{i}(x)\right] \rightarrow r_{x}$ implies $O\left[W_{i} V_{i}(x)\right] \rightarrow r_{x}$. The new sequence $\left[W_{i} V_{i}\right](x)$ being equicontinuous and uniformly bounded, Ascoli's theorem applies and it has a limit $y$ in $K[x]$.

${ }^{5}$ The terminology and notation in the remainder of this section are those of [3, Chapter II]. 
6. The foregoing is not sufficient to obtain a unique constant function in $K[x]$.

EXAMPLE 6. Let $S=\left[\begin{array}{ll}0 & 1\end{array}\right], F=X=C(S)$. Let $G$ consist of the transformations indexed by $S$ :

$$
\left[T_{s}(x)\right](t)=x(s) \quad(s \text { arbitrarily chosen in } S) .
$$

Each $x$ satisfies $A_{1}$ and $A_{2}^{\prime}$ since for any $V \in G_{1}$ the oscillation of $V(x)$ over any neighborhood is bounded by that of $x$. But for each value in the range of $x$ there is a constant function in $K[x]$.

Thus it is necessary to use some additional condition to ensure uniqueness. The following theorem is an example of a restriction which provides uniqueness of the constant function in $K[x]$.

THEOREм 6.1. Let $G$ be bounded by $k$ and let the constant functions be fixed under $G$. Let $x$ satisfy the following condition:

$\left(\mathrm{B}_{1}\right)$ For each $T \in G$ and $\epsilon>0$ there is a $\delta>0$ such that if $V \in G_{1}$ and $O[V(x)]<\delta$, then for each $t \in S$ there is a $t^{\prime}$ with

$$
\left|[V T(x)](t)-[V(x)]\left(t^{\prime}\right)\right|<\epsilon .
$$

Then $K[x]$ contains at most one constant function.

Proof. Suppose $\bar{x}$ and $\bar{y}$ are constant functions in $K[x]$. Take $\epsilon>0$. There is a

$$
V \in G_{1}, V=\sum_{1}^{n} \lambda_{i} T_{i}
$$

with

$$
\|V(x)-x\|<\epsilon / 3 k
$$

For each $T_{i}$, let $\epsilon / 3$ be the $\epsilon$ and $\delta_{i}$ be the corresponding $\delta$ of $B_{1}$. Take $\mathbb{W} \in G_{1}$ such that

$$
\|\mathbb{W}(x)-\bar{y}\|<\min \left\{\epsilon / 3, \delta_{i} / 2\right\} \quad(i=1,2, \cdots n) .
$$

Then $O[W(x)]<\delta_{i}$ for each $i$. For an arbitrarily fixed $t$ and for each $T_{i}$ there is a $t_{i}^{\prime}$ such that 


$$
\left|\left[W T_{i}(x)\right](t)-[W(x)]\left(t_{i}^{\prime}\right)\right|<\epsilon / 3,
$$

But

$$
\left|[W(x)]\left(t_{i}^{\prime}\right)-\bar{y}(t)\right|=\left|[W(x)]\left(t_{i}^{\prime}\right)-\bar{y}\left(t_{i}^{\prime}\right)\right| \leqq\|W(x)-\bar{y}\|<\epsilon / 3 .
$$

Hence by addition,

$$
\left|\left[W T_{i}(x)\right](t)-\bar{y}(t)\right|<2 \epsilon / 3 \text {. }
$$

Since $t$ was arbitrarily chosen,

$$
\left\|W T_{i}(x)-\bar{y}\right\| \leqq 2 \epsilon / 3
$$

and

$$
\|W V(x)-\bar{y}\|=\left\|\mathbb{W} \lambda_{i} T_{i}(x)-\sum \lambda_{i} \bar{y}\right\| \leqq \sum \lambda_{i}\left\|W T_{i}(x)-\bar{y}\right\| \leqq 2 \epsilon / 3 .
$$

But

$$
\|\mathbb{W} V(x)-\bar{x}\|=\|W V(x)-W(\bar{x})\| \leqq\|W\| \cdot\|V(x)-\bar{x}\|<\epsilon / 3 .
$$

Adding again, we get $\|\bar{x}-\bar{y}\|<\epsilon$, whence $\bar{x}=\bar{y}$.

It is possible to find examples of the von Neumann case which do not satisfy $\mathrm{B}_{1}$, when $S$ is a non-abelian finite group in the discrete topology. A theorem of Eberlein [6, p. 230] applies more directly, and although it is not of the type we are seeking, we state it here for completeness. ${ }^{6}$

THEOREM 6.2 [Eberlein]. Let $G^{1}$ and $G^{2}$ be bounded semi-groups that commute elementwise (that is, if $T_{1} \in G^{1}$ and $T_{2} \in G^{2}$ then $T_{1} T_{2}=T_{2} T_{1}$ ) and let every fixed point of $G^{1}$ be a fixed point of $G^{2}$, and conversely. Then the fixed points of $K^{1}[x]$ and $K^{2}[x]$ reduce to a common, unique fixed point.

In the von Neumann construction, the $G^{1}$ and $G^{2}$ are the transformations by multiplication on the left and right in $S$. Since $G^{1}$ and $G^{2}$ each satisfy $\mathrm{A}_{1}$ and $A_{2}$, the constant functions are the only fixed points for both, and for each $x$, $K^{1}[x]$ and $K^{2}[x]$ have one in common.

Alaoglu and Birkhoff give the following theorem [1, p.300]: If $G$ is bounded, and if for any $x$ the means of its transforms have the Moore-Smith property, then

\footnotetext{
${ }^{6}$ With the exception of Theorems 6.4 and 6.7 , the actual nature of $X$, other than its
} being a normed linear space, is not considered in the remainder of this section. 
$K[x]$ contains not more than one fixed point. With their definition of order for $G_{1}(x)$, this is equivalent to: Given any $x \in X, G$ bounded; if, for any $V, \mathbb{W}$ in $G_{1}$ there exist $V^{\prime}, W^{\prime}$ in $G_{1}$ such that

$$
V^{\prime} V(x)=\mathbb{W}^{\prime} \mathbb{W}(x),
$$

then $K[x]$ contains not more than one fixed point. The hypothesis here can be weakened a little, since only an arbitrarily close approach is needed, rather than equality.

THEOREM 6.3. If $G$ is bounded by $k$, and $x$ satisfies:

$\left(B_{2}\right)$ for any $\epsilon>0$ and any $V, \mathbb{W}$ in $G_{1}$ there exist $V^{\prime}, W^{\prime}$ in $G_{1}$ such that

$$
\left\|V^{\prime} V(x)-W^{\prime} W(x)\right\|<\epsilon
$$

then $K[x]$ contains not more than one fixed point.

Proof. Let $\bar{y}$ and $\bar{z}$ be fixed points in $K[x]$. Take $\epsilon>0$. Then there are $V, \mathbb{W}$ in $G_{1}$ such that

$$
\|\bar{y}-V(x)\|<\epsilon / 3 k \text { and }\|W(x)-\bar{z}\|<\epsilon / 3 k .
$$

By the hypothesis, there exist $V^{\prime}, W^{\prime}$ in $G_{1}$ such that

$$
\left\|V^{\prime} V(x)-\mathbb{W}^{\prime} \mathbb{W}(x)\right\|<\epsilon / 3
$$

But

$$
\left\|\bar{y}-V^{\prime} V(x)\right\|=\left\|V^{\prime}(\bar{y})-V^{\prime} V(x)\right\| \leqq\left\|V^{\prime}\right\| \cdot\|\bar{y}-V(x)\|<\epsilon / 3 .
$$

Similarly,

$$
\|\mathbb{W} W(x)-\bar{z}\|<\epsilon / 3
$$

Hence, by addition,

$$
\|\bar{y}-\bar{z}\|<\epsilon
$$

Since $\epsilon>0$ is arbitrary, $y=z$.

COROLLARY 6.1. If $G$ is bounded and abelian with respect to a given $x$, then $K[x]$ contains not more than one fixed point. 
Proof. Clearly, $x$ satisfies $\mathrm{B}_{2}$ with $V^{\prime}=W$ and $W^{\prime}=V$.

If the constant functions are fixed points, $\mathrm{B}_{2}$ can be further weakened to get a similar conclusion about constant functions.

THEOREM 6.4. If $G$ is bounded by $k$ and the constant functions are fixed points, and $x$ satisfies the following condition:

$\left(\mathrm{B}_{3}\right)$ for any $\epsilon>0$ and any $V, \mathbb{W}$, in $G_{1}$, there are $V^{\prime}, W^{\prime}$, in $G_{1}$ and $t_{1}$, $t_{2}$ in $S$ such that

$$
\left|\left[V^{\circ} V(x)\right]\left(t_{1}\right)-\left[\mathbb{W}^{\prime} \mathbb{W}(x)\right]\left(t_{2}\right)\right|<\epsilon,
$$

then $K[x]$ contains not more than one constant function.

Proof. As in Theorem 6.3 we obtain $V, W$, such that

$$
\left\|\bar{y}-V^{\prime} V(x)\right\|<\epsilon / 3 \text { and }\left\|W^{\prime} W(x)-\bar{z}\right\|<\epsilon / 3
$$

for any $V^{\prime}, \mathbb{W}^{\prime}$ in $G_{1}$. From the hypothesis there exist $V^{\prime}, \mathbb{W}^{\prime}, t_{1}, t_{2}$, such that

$$
\left|\left[V^{\prime} V(x)\right]\left(t_{1}\right)-\left[W^{\prime} W(x)\right]\left(t_{2}\right)\right|<\epsilon / 3 \text {. }
$$

For any $t \in S$, we have from the first inequalities,

$$
\left|\bar{y}(t)-\left[V^{\prime} V(x)\right]\left(t_{1}\right)\right|=\left|\bar{y}\left(t_{1}\right)-\left[V^{\prime} V(x)\right]\left(t_{1}\right)\right| \leqq\left\|\bar{y}-V^{\prime} V(x)\right\|<\epsilon / 3
$$

and

$\left|\left[\mathbb{W}^{\prime} \mathbb{W}(x)\right]\left(t_{2}\right)-\bar{z}(t)\right|=\left|\left[\mathbb{W}^{\prime} \mathbb{W}(x)\right]\left(t_{2}\right)-\bar{z}\left(t_{2}\right)\right| \leqq\left\|W^{\prime} W^{\prime}(x)-\bar{z}\right\|<\epsilon / 3$.

Adding the three last inequalities we get our conclusion.

It can be seen that the von Neumann case satisfies $B_{3}$ although to show it would amount to a proof of the theorem.

With a somewhat stronger conclusion of Theorem $6.3, B_{2}$ becomes a necessary condition.

THE оRем 6.5. Let $G$ be bounded by $k$. For a given $x \in X$, suppose that $K[x]$ and, for any $V \in G_{1}, K[V(x)]$ each have just one fixed point. Then $\mathrm{B}_{2}$ holds.

Proof. Take any $V, \mathbb{W}$ in $G_{1}, \epsilon>0$. We have shown in Lemma 4.1 that 
$K[V(x)]$ and $K[I f(x)]$ are both contained in $K[x]$. Hence by the hypothesis they contain a common, unique fixed point $\bar{x}$. There exist $V^{\prime}, W^{\prime}$ in $G_{1}$ such that

$$
\left\|V^{\prime} V(x)-\bar{x}\right\|<\epsilon / 2 \text { and }\left\|\bar{x}-\mathbb{W}^{\prime} \mathbb{W}(x)\right\|<\epsilon / 2 \text {, }
$$

whence the conclusion follows by addition.

Combining these results we get:

Corollary 6.2. If $G$ is bounded, and for each $x \in F, K[x]$ contains $a$ fixed point and $V(x) \in F$ for any $V \in G_{1}$, then a necessary and sufficient condition that it be unique is that for each $x \in F, \mathrm{~B}_{2}$ holds.

The next condition that we consider, while stronger that $B_{2}$, has the advantage of relating to a $T \in G$ and a $\mathbb{W} \in G_{1}$ instead of two transformations in $G_{1}$. It also has an affinity to Theorem 6.8 , since it exhibits a sort of commutativity.

THEOREM 6.6. Let $G$ be bounded. If $x \in X$ satisfies the following condition:

$\left(\mathrm{B}_{4}\right)$ for each $T \in G$ and $W \in G_{1}$ and any $\epsilon>0$, there is a $Z \in G_{1}$ such that

$$
\|\mathbb{W} T(x)-Z \mathbb{W}(x)\|<\epsilon
$$

then $\mathrm{B}_{2}$ holds for $x$.

Proof. Take

$$
V, \mathbb{W} \text { in } G_{1}, \epsilon>0, V=\sum_{1}^{n} \lambda_{i} T_{i} \quad\left(T_{i} \in G\right) .
$$

For each $T_{i}$ we have a $Z_{i} \in G_{1}$ such that

$$
\left\|W T_{i}(x)-Z_{i} W(x)\right\|<\epsilon \text {. }
$$

Hence

$$
\begin{aligned}
& \left\|\left[\mathbb{W}\left(\sum \lambda_{i} T_{i}\right)\right](x)-\left[\left(\sum \lambda_{i} Z_{i}\right) \mathbb{W}\right](x)\right\| \\
& \qquad \sum\left[\lambda_{i}\left\|\mathbb{W} T_{i}(x)-Z_{i} \mathbb{W}^{\prime}(x)\right\|\right]<\sum \lambda_{i} \epsilon=\epsilon
\end{aligned}
$$

Letting 


$$
W=V^{\prime}, \quad \sum_{1}^{n} \lambda_{i} Z_{i}=W^{\prime}
$$

we have

$$
\left\|V^{\prime} V(x)-W^{\prime} W(x)\right\|<\epsilon
$$

To summarize the main results thus far:

The OREM 6.7. Let $G$ be bounded by $k, S$ compact. Suppose for each $x \in F$ :

(1) $x$ is lower or upper semi-continuous,

(2) $x$ satisfies $\mathrm{A}_{1}$ and $\mathrm{A}_{2}$,

(3) for each $V \in G_{1}, V(x) \in F$ and each $V(x)$ has the same kind of semicontinuity as $x$,

(4) $x$ satisfies $\mathrm{B}_{1}$ or $\mathrm{B}_{2}$ or $\mathrm{B}_{3}$ or $\mathrm{B}_{4}$, or $G$ is abelian over $x$,

(5) F contains a nonzero constant function.

Then:

(I) There exists a unique bounded linear functional over $X$, invariant with respect to $G$, and with $x^{*}(e)=1$,

(II) $\mathrm{P}_{1}$ holds over $F$,

(III) if for each $x \in F, x$ is lower semi-continuous, then $\mathrm{P}_{2}$ holds over $F$.

Proof. By (1), (2), and (3), $x$ satisfies the conditions for Theorem 5 . Hence there is a constant function in $K[x]$, for each $x \in F$. By Lemma 5.1 the constant function in $F$ is a fixed point, and hence by linearity all constant functions in $X$ are fixed points. With (4) each $x \in F$ satisfies the appropriate theorem in $\$ 6$ to provide the uniqueness of the constant function. With $(3)$ and (5) the conditions for Theorem 4.1 are satisfied, yielding the normalized invariant functional, and Theorem 4.2 makes it unique. Theorems 5 and 4.1 also yield II and III of our conclusion.

CoROLLARY 6.3. If $F=X \subset C(S)$ and (2) and (4) hold, then there is an invariant integral over $X$. If $S$ is also uniform, $\mathrm{A}_{2}$ may be replaced by $\mathrm{A}_{2}^{\prime}$ in (2).

In concluding this section we shall change our viewpoint some what. Suppose, in Theorem 6.7, all conditions up to and including (3) are satisfied, and 
suppose in addition there exists any nonzero invariant bounded linear functional $y^{*}$ over $X$. By Theorem 5 there is a constant function in each $K[x]$, for each $x \in F$. Hence by Lemma 4.2,

$$
y^{*}(e)=a \neq 0 \text {. }
$$

For if $y^{*}(e)=0$, then $y^{*}(\bar{x})=0$ for any constant function, and therefore everywhere on $F$; thus by linearity and continuity $y^{*}(\bar{x})=0$ everywhere on $X$. So we can obtain a bounded linear functional

$$
x^{*}=\frac{1}{a} y^{*}
$$

satisfying $\mathrm{I}$ and $\mathrm{N}$, and by Theorem 4.2 it is unique. Hence any additional condition which produces a nonzero invariant bounded linear functional replaces (4) and (5) in Theorem 6.7. For example, in a paper by B. Yood [10], several conditions are given, under each of which such a functional exists over a normed linear space $X$, provided $G$ is bounded (actually less than this is assumed) and satisfies the following necessary condition:

(D) There exists an $x \in X$ such that $\inf _{T \in G}\|T(x)\|>0$.

(This condition is fulfilled by the constant functions in our case.) Some of the results may be summarized:

A nonzero invariant bounded linear functional exists if $G$ is bounded, satisfies condition (D), and

1) $G$ is an abelian semi-group, or

2) $G$ is a solvable group, or

3) $G$ is a finite group.

The first is covered in Theorem 6.7. The last uses a strong group property (existence of inverse) and cannot be extended to semi-groups. However, at his suggestion we were able to extend the second to semi-groups, using a definition somewhat similar to one of M. M. Day [4, p. 285].

DEFINITION 6.1. $H \in G$ is a left commutator sub-semi-group of $G$ if:

(1) $H$ is a semi-group,

(2) for each $T, U$, in $G$ there is a $V \in H$ such that $T U=V U T$.

DEFINITION 6.2. $G$ is a left solvable semi-group if there is a sequence 


$$
G=G^{0} \supset G^{1} \supset G^{2} \supset \ldots \supset G^{n}
$$

such that $G^{i+1}$ is a left commutator sub-semi-group for $G^{i}$, and $G^{n}$ is abelian.

THEOREM 6.8. Let $G=G^{0}$ be a left solvable semi-group. For each $i$, let $G^{i}$ satisfy the following relaxed boundedness condition: There is a $k(i)>0$ such that for each $V \in G_{1}^{i}$ there is a $W \in G_{1}^{i}$ with

$$
\|\mathbb{W}\| \leqq k(i) \text { and }\|\mathbb{W} V\| \leqq k(i) \text {. }
$$

Assume further that condition (D) is satisfied. Then there is a nonzero invariant bounded linear functional over $X$.

The proof essentially follows the lines of Professor Yood's paper.

7. In section 5, and hence in Theorem 6.7 and its Corollary 6.3, the compactness of $S$ played an important part. This can be relaxed at the expense of limiting $F$. In this section, $S$ will be a noncompact Hausdorff space. For any $x \in X$ the following conditions are equivalent. ${ }^{7}$

$\left(\mathrm{L}_{1}\right)$ There is a finite number $r_{x}$ such that for any $\epsilon>0$ there is a compact set $R_{\epsilon} \subset S$ with $\left|r_{x}-x(t)\right|<\epsilon$ for all $t \in\left(S-R_{\epsilon}\right)$. ( $\left(S-R_{\epsilon}=\right.$ complement of $R_{\epsilon}$ in $\left.S.\right)$

$\left(\mathrm{L}_{2}\right) \quad$ For each $\epsilon>0$ there is a compact set $R_{\epsilon} \subset S$ such that $O[x]<\epsilon$ on $S-R_{\epsilon}$.

It is clear that $\mathrm{L}_{1}$ implies $\mathrm{L}_{2}$. For the converse, take $\epsilon_{i}=1 / i(i=1,2, \ldots)$. For $i=1$, let $S_{1}=R_{1}$ be the corresponding compact set under the hypothesis. Let $Z_{1}=S-S_{1}$. Since $S$ is not compact, $Z_{1} \neq \varnothing$. Select a $t_{1} \in Z_{1}$. Then for all $t \in Z_{1},\left|x(t)-x\left(t_{1}\right)\right|<1=\epsilon_{1}$. For $i=2$, let $R_{2}$ be the corresponding compact set. Take $S_{2}=S_{1}+R_{2}$ (set union). $S_{2}$ is compact. Let $Z_{2}=S \neq \varnothing$. Select $t_{2} \in Z_{2} . Z_{2}=S-S_{2}=S-\left(S_{1}+R_{2}\right) \subset S-S_{1}=Z_{1}$. Hence $Z_{2} \subset Z_{1}$ and $Z_{2} \subset S-R_{2}$. From the second inclusion, $\left|x(t)-x\left(t_{2}\right)\right|<1 / 2$ for all $t \in Z_{2}$. We now proceed with an inductive construction. Assume that $S_{i}, Z_{i}=S-S_{i}$, and $t_{i} \in S$ have been chosen for each $i \leqq n$, satisfying the four conditions:

(1) $S_{i}$ is compact,

(2) $t_{i} \in Z_{i}$,

(3) $Z_{n} \subset Z_{n-1} \subset \cdots \subset Z$,

(4) $\left|x(t)-x\left(t_{i}\right)\right|<1 / i$ all $t \in Z_{i}$.

7 If we define $O[z]$ over $S^{\prime} C S$ as $\sup \left|z\left(t_{1}\right)-z\left(t_{2}\right)\right|\left(t_{1}, t_{2} \in S^{\prime}\right)$, then the equivalence of $L_{1}$ and $L_{2}$ holds for any complex-valued function $z$ defined on $S$. 
It then follows that for each $i$ and $p=0,1, \cdots, n-1$,

$$
\left|x\left(t_{i}\right)-x\left(t_{i+p}\right)\right|<\frac{1}{i} .
$$

For $i=n+1$, let $R_{n+1}$ be the corresponding compact set. Take $S_{n+1}=S_{n}+R_{n+1}$.

(1) $S_{n+1}$ is compact. Therefore we may take

(2) $t_{n+1}$ in $Z_{n+1}=S-S_{n+1} \neq \varnothing$.

(3) $Z_{n+1} \subset Z_{n}$ and $Z_{n+1} \subset S-R_{n+1}$ (as with $Z_{2}$ ), and therefore

(4) $\left|x(t)-x\left(t_{n+1}\right)\right|<1 /(n+1)$ for all $t \in Z_{n+1}$.

Thus we define a sequence of real numbers $\left\{r_{i}\right\}=\left\{x\left(t_{i}\right)\right\}$ such that for any $i$ and any $p \geqq 0$,

$$
\left|r_{i}-r_{i+p}\right|<\frac{1}{i}
$$

$r_{i}$ is then a convergent sequence. Call its limit $r_{x}$. Take any $\epsilon>0$. For $i$ sufficiently great we have

$$
\left|x\left(t_{i}\right)-r_{x}\right|<\frac{\epsilon}{2} \text { and } \frac{1}{i}<\frac{\epsilon}{2} .
$$

Then for any $t$ in $Z_{i}=S-S_{i}$,

$$
\left|x(t)-r_{x}\right| \leqq\left|x(t)-x\left(t_{1}\right)\right|+\left|x\left(t_{i}\right)-r_{x}\right|<\frac{\epsilon}{2}+\frac{\epsilon}{2}=\epsilon .
$$

With $S_{i}=R_{\epsilon},\left(\mathrm{L}_{1}\right)$ is established. We note also that for each $x, r_{x}$ is unique. Suppose $r_{x}$ and $s_{x}$ are limits for $x$. Then for any $\epsilon>0$ there are compact sets $R_{\epsilon}$ and $S_{\epsilon}$, such that

$$
\left|x(t)-r_{x}\right|<\frac{\epsilon}{2} \quad \text { for } t \text { in } S-R_{\epsilon},
$$

and

$$
\left|x(t)-s_{x}\right|<\frac{\epsilon}{2} \quad \text { for } t \text { in } S-S_{\epsilon} \text {. }
$$

But $R_{\epsilon}+S_{\epsilon}$ is compact and therefore $Z=S-\left(R_{\epsilon}+S_{\epsilon}\right)$ is not empty. Hence 
take $t \in Z$. Both inequalities hold for this $t$ and we get

$$
\left|r_{x}-s_{x}\right| \leqq\left|r_{x}-x(t)\right|+\left|x(t)-s_{x}\right|<\epsilon
$$

So $r_{x}=s_{x}$.

We now embed $S$ in a compact space $\underline{S}$ by the addition of an ideal point $z$. The topology of $\underline{S}$ is given by defining its closed sets as follows:

For each set $C$ closed in $S, C+z$ is closed in $\underline{S}$. If $C$ is also compact, then $C$ is closed in $\underline{S}$.

REMARK. With $S$ embedded thus, the relative topology of $S$ as a subset of $\underline{S}$ is equivalent to its original topology. Since $S$ is a Hausdorff space, compact sets are closed in $S$. However, unless $S$ is also locally compact, we cannot conclude that $\underline{S}$ is Hausdorff $[7, \mathrm{pp} .20-23]$.

For the remainder of this section we shall assume that $L_{1}$ is satisfied by each $x \in X$. Corresponding to each $x$ we define a function $\underline{x}$ over $\underline{S}$ :

$$
\begin{array}{ll}
\underline{x}(t)=x(t) & (t \in S), \\
\underline{x}(z)=r_{x} . &
\end{array}
$$

Call the collection of functions so formed $\underline{X}$.

Lemma 7.1. If $F \subset C(S)$ then $\underline{X} \subset C(\underline{S})$.

Proof. From the topology defined in $\$ 2$, convergence in $B(S)$ means uniform convergence. Hence $X$ is contained in $C(S)$ if $F$ is. Now take any $\underline{x} \in \underline{X}$. By definition and the above remark it is continuous at every point in $S=\underline{S}-z$. We need only show that it is continuous at $z$. For any $\epsilon>0$, there is a compact, (and hence closed in $S$ ) set $C \subset S$, such that

$$
|\underline{x}(z)-\underline{x}(t)|=\left|r_{x}-x(t)\right|<\epsilon
$$

for all $t \in S-C$, and so for all $t \in \underline{S}-C$. But $C$ is also closed in $\underline{S}$, whence $\underline{S}-C$ is open in $\underline{S}$. Since it contains $z, \underline{x}$ is thereby continuous at $z$, and $\underline{X} \subset C(\underline{S})$.

Suppose we have a $G$ as before. Define $\underline{G}=\underline{T}$ operating on $\underline{X}$ by:

$$
\underline{T}(\underline{x})=\underline{T(x)}
$$

Then by a series of trivial verifications we get: 


\section{LEMMA 7.2.}

(1) $\underline{G} \subset B[\underline{X}]$, and

(2) the mappings $i(x)=\underline{x}$ and $j(T)=\underline{T}$ are isomorphic and isometric.

We are now in a position to enquire to what extent conditions on $G$ and $X$ are equivalent to those on $\underline{G}$ and $\underline{X}$; for any functional on one defines a functional on the other with the same properties, according to the isometries and isomorphisms. We have the following:

THEOREM 7.1.

(1) $\mathrm{A}_{1}$ holds for $G$ and $x$ if it holds for $\underline{G}$ and $\underline{x}$;

(2) $\mathrm{A}_{2}$ holds for $G$ and $x$ if and only if it holds for $\underline{G}$ and $\underline{x}$.

Proof. For $A_{1}$ we will first show that $\inf _{t \in S} x(t)=\inf _{t \in \underline{S}} \underline{x}(t)$ for all $x \in X$. The relationships on the sups go the same way. Since $\overline{S \subset \underline{S}}$, we have, for any $x \in X$,

$$
\inf _{t \in \underline{S}} \underline{x}(t) \leqq \inf _{t \in S} \underline{x}(t)=\inf _{t \in S} x(t)
$$

But for all $t \in S$,

$$
\underline{x}(t)=x(t) \geqq \inf _{t \in S} x(t) .
$$

For any $\epsilon>0$, there is a $t_{\epsilon}$ in $S$ such that

$$
\underline{x}(z)>\underline{x}\left(t_{\epsilon}\right)-\epsilon \geqq \inf _{t \in S} x(t)-\epsilon \text {. }
$$

Hence for all $t \in \underline{S}$,

$$
\underline{x}(t) \geqq \inf _{t \in S} x(t),
$$

and thus

$$
\inf _{t \in \underline{S}} \underline{x}(t) \geqq \inf _{t \in S} x(t) .
$$

Combining this with (1) above we get:

$$
\inf _{t \in \underline{S}} \underline{x}(t)=\inf _{t \in S} x(t) .
$$


Now suppose that for each $t_{0} \in \underline{S}$,

$$
\inf _{\underline{V} \in \underline{G}_{1}}[\underline{V}(\underline{x})]\left(t_{0}\right)=\inf _{t \in \underline{S}} \underline{x}(t) .
$$

Then the corresponding equality holds for $x$ and $G$ and any $t_{0} \in S$, by the inclusion of $S$ in $\underline{S}$ and (2) above.

For $\mathrm{A}_{2}$ we have by the isometry of the mapping $i(x)=\underline{x}$ that

$$
\left\|W_{n}(x)-y\right\| \rightarrow 0
$$

if and only if

$$
\left\|\underline{W}_{n}-\underline{y}\right\| \rightarrow 0 \text {. }
$$

Hence convergence and closure in $K[x]$ correspond to convergence and closure in $K[x]$.

As a counter-example to the converse of part ( 1 ) of the last theorem, take $S$ as the real line, $X$ the bounded functions over $S$ which approach equal limits to the right and left, and $G=\left\{T_{a}\right\}, a$ in $S$, with

$$
\left[T_{a}(x)\right](t)=x(t+a) \text {. }
$$

Clearly $\mathrm{A}_{1}$ is satisfied for $S, X$, and $G$, but for any $\underline{V} \in \underline{G}_{1}$,

$$
[\underline{V}(\underline{x})](z)=\underline{x}(z)=\lim _{|x| \rightarrow \infty} x(t) .
$$

In $\$ 6$ we were concerned with the conditions limiting the number of fixed points in $K[x]$. Our arguments did not depend on the compactness of $S$, and we have shown that convergence and closure correspond. There remains the following:

THEOREM 7.2. A point $x$ is a fixed point under $G_{1}$ if and only if $\underline{x}$ is a fixed point under $\underline{G}_{1}$.

Proof. Let $V(x)=x$. Then

$$
[\underline{V}(\underline{x})](t)=\underline{x}(t)
$$

for all $t \in S$. For a given $\epsilon>0$, let $R_{\epsilon}, R_{\epsilon}^{\prime}$ be the compact sets of $\mathrm{L}_{1}$ for $x$ and $V(x)$ respectively. As before, $S-\left(R_{\epsilon}+R_{\epsilon}^{\prime}\right)$ is not empty, and we can choose 
a $t_{\epsilon}$ such that

$$
\left|\underline{x}(z)-\underline{x}\left(t_{\epsilon}\right)\right|=\left|r_{x}-x\left(t_{\epsilon}\right)\right|<\epsilon,
$$

and

$$
\begin{aligned}
& \left|[\underline{V}(\underline{x})](z)-\underline{x}\left(t_{\epsilon}\right)\right| \\
& \quad=\left|[\underline{V}(\underline{x})](z)-[\underline{V}(\underline{x})]\left(t_{\epsilon}\right)\right|=\left|r_{V x}-[V(x)](t \epsilon)\right|<\epsilon .
\end{aligned}
$$

Combining these inequalities, we get

$$
|[\underline{V}(\underline{x})](z)-\underline{x}(z)| \leqq\left|[\underline{V}(\underline{x})](z)-\underline{x}\left(t_{\epsilon}\right)\right|+\left|\underline{x}\left(t_{\epsilon}\right)-\underline{x}(z)\right|<2 \epsilon .
$$

Hence, by the arbitrary nature of $\epsilon>0$, we get equality at $z$ and thus for all $t \in \underline{S}$. The converse result follows by inclusion.

This completes the comparison of the main results, concerning semi-continuous functions over a compact space, with a restricted class of functions over a Hausdorff space. We now give one example (or rather, a family of examples ) of some interest.

EXAMPLE 7. Let $S$ be the real line in the usual topology. Let $F=X$ be the collection of continuous functions which approach equal limits to the left and right. Let $\phi$ be any homeomorphism of $S$ onto the open interval $(0,1) ; \phi$ can be extended to map $\underline{S}$ onto $R=[0,1]$ with 0 and 1 identified. " This is a compact topological group under addition $\bmod 1$. If $Y=C(R)$ we have the group $G=\left\{T_{a}\right\}$ of transformations on $Y$ :

$$
\left[T_{a}(y)\right](r)=y(r+a) \quad(0 \leqq a<1),
$$

with $r+a$ being taken mod 1 . Now $\phi$ induces a transformation $\Phi: \underline{X} \rightarrow Y$, where

$$
[\Phi(x)](r)=\underline{x}\left(\phi^{-1} r\right) .
$$

For each such mapping $\phi$, we have a group $G_{\phi}=\left\{U_{a}\right\}$ of transformations on $X$ as follows:

$$
U_{a}(x)=\left[i^{-1} \Phi^{-1} T_{a} \Phi i\right](x)
$$

$G_{\phi}$ will have whatever properties $G$ has toward the construction of a unique invariant integral $x_{\phi}^{*}$. It is easy to see that

$$
x_{\phi}^{*}(x)=y^{*}(\Phi \underline{x}),
$$


where $y^{*}$ is the (von Neumann) invariant integral over $Y$.

\section{REFERENCES}

1. L. Alaoglu and G. Birkhoff, General ergodic theorems, Ann. of Math. (2) 41 (1940), 293 - 309.

2. S. Banach, Theorie des operations lineaires, Warsaw, 1933.

3. N. Bourbaki, Topologie generale, Paris, 1940 and 1949.

4. M. M. Day, Means for the bounded functions and ergodicity of the bounded representations of semi-groups, Trans. Amer. Math. Soc. 69 (1950), 276-291.

5. J. Dixmier, Moyennes invariantes dans le semi-groupes, Acta Sci. Math. Szeged 12 (1950), $213-227$.

6. W. F. Eberlein, Abstract ergodic theorems and weak almost periodic functions, Trans. Amer. Math. Soc. 67 (1949), 217-240.

7. S. Lefschetz, Algebraic topology, Amer. Math. Soc. Colloquium Publications, New York, 1942.

8. L. Pontrjagin, Topological groups, Princeton Univ. Press, Princeton, 1939.

9. M. H. Stone, Applications of the theory of boolean rings to general topology, Trans. Amer. Math. Soc. 41 (1937), 375-481.

10. B. Yood, On fixed points for semi-groups of linear operators, Proc. Amer. Math. Soc. 2 (1951), 225- 233.

\section{University of Connecticut}




\title{
PACIFIC JOURNAL OF MATHEMATICS
}

\section{EDITORS}

\author{
M. M. SCHIFFER* \\ Stanf ord University \\ Stanf ord, California \\ E. HewitT \\ University of Washington \\ Seattle 5, Washington
}

\author{
P. P. DILWCR TH \\ California Institute of Technology \\ Pasadena 4, California \\ E. F. BECKENBACH** \\ University of California \\ Los Angeles 24, California
}

\section{ASSOCIATE EDITORS}

$\begin{array}{ll}\text { H. BUSEMANN } & \text { P. R. HALMOS } \\ \text { HERDERT FF.DERER } & \text { HEINZ HOPF } \\ \text { MARSH ALLL IHALL } & \text { R. D. JAMES }\end{array}$

MARSH AL.L II ALL

\author{
R. D. JAMES
}
BORGE JESSEN
PAUL LÉVY
GEORGE PÓLYA

\author{
J. J. STOKER \\ E. G. STRAUS
}
KÖSAKU YOSIDA

\section{SPONSORS}

UNIVERSITY OF BRITISH COLUMBIA CAI IFORNIA ENSTITUTE OF TECHNOLOGY UNIVERSITY OF CALIFORNIA, BERKELEY UNIVERSITY OF CALIFORNIA, DAVIS UNIVERSITY OF CALIFORNIA, LOS ANGELES UNIVERSITY OF CALIFORNI A, SANTA BARBARA UNIVERSITY OF NEVADA OREGON STATE COLLEGE UNIVERSITY OF OREGON
UNIVERSITY OF SOU THERN CALIFORNIA STANFORD RESE.ARCH INSTITUTE STANFORD UNIVERSITY WASHING TON STATE COLLEGE UNIVERSITY OF WASHINGTON

AMERICAN MATHEMATICAL SOCIETY HUGHES AIRCRAFT COMPANY SHELL DEVELOPMENT COMPANY

* To be succeeded in 1955, by H.L. Royden, Stanford University, Stanford, California.

${ }^{* *}$ To be succeeded in 1955, by E.G. Straus, University of California, Los Angeles 24, Calif.

\author{
Vari-Type Composition by \\ Elaine Barth
}

Printed in the United States of America by

Edwards Brothers, Inc., Ann Arbor, Michigan

UNIVERSITY OF CALIFORNIA PRESS * BERKELEY AND LOS ANGELES COPYRIGHT 1954 BY PACIFIC JOURNAL OF MATHEMATICS 


\section{Pacific Journal of Mathematics}

\section{Vol. 4, No. 1 \\ May, 1954}

Hugh D. Brunk, On the growth of functions having poles or zeros on the positive real axis ................................. 1

J. Copping, Application of a theorem of Pólya to the solution of an infinite

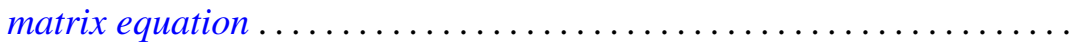

James Richard Jackson, On the existence problem of linear programming ................................... 29

Victor Klee, Invariant extension of linear functionals ............... 37

Shu-Teh Chen Moy, Characterizations of conditional expectation as a transformation on function spaces ..................... 47

Hukukane Nikaidô, On von Neumann's minimax theorem .............. 65

Gordon Marshall Petersen, Methods of summation .................. 73

G. Power, Some perturbed electrostatic fields .................. 79

Murray Harold Protter, The two noncharacteristic problem with data partly

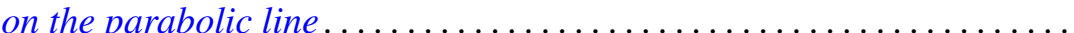

S. E. Rauch, Mapping properties of Cesàro sums of order two of the geometric series........................................... 109

Gerson B. Robison, Invariant integrals over a class of Banach spaces . . . . 123

Richard Steven Varga, Eigenvalues of circulant matrices .............. 151 